\title{
Study on the mechanism of Indigo naturalis and its two main compounds (indigo and indirubin) in the treatment of ulcerative colitis based on TLR4/MyD88/NF-KB pathway and intestinal microorganisms
}

Qi-yue Yang

Hosiptal of Chengdu University of traditinal Chinese Medicine

Ya-nan He

Chengdu University of Traditional Chinese Medicine

Le-le Ma

Chengdu University of Traditional Chinese Medicine

\section{Run-chun Xu}

Chengdu University of Traditional Chinese Medicine

Nan Li

Chengdu University of Traditional Chinese Medicine

Li Han

Chengdu University of Traditional Chinese Medicine

Xiang-bo Yang

Ya'an Xunkang Pharmacuetical Co., Ltd

\section{Fang Wang}

Jiangxi University of Traditional Chinese Medicine

Dingkun Zhang (D 465790643@qq.com)

Chengdu University of Traditional Chinese Medicine https://orcid.org/0000-0001-6746-3454

\section{Research}

Keywords: Indigo naturalis, indigo, indirubin, ulcerative colitis, gut microbial

Posted Date: May 29th, 2020

DOI: https://doi.org/10.21203/rs.3.rs-31454/v1

License: (c) (i) This work is licensed under a Creative Commons Attribution 4.0 International License.

Read Full License 


\section{Abstract}

Background: Indigo naturalis is a natural dye extracted from plants and has a good anti-inflammatory effect. Clinical studies have shown that it can improve ulcerative colitis (UC), but the active constituents and the mechanism are unclear.

Methods: The anti-UC activity of Indigo naturalis and its two main compounds (indigo and indirubin) were investigated in dextran sulfate sodium (DSS)-induced UC mice. Indigo naturalis, indigo and indirubin were administrated to DSS-induced UC rats by oral gavage for 1 weeks. The anti-UC effect was evaluated by pathological section, inflammatory cytokine production, western blotting, and gut microbiota analysis via 16S rRNA sequencing.

Results: Indigo naturalis, indigo and indirubin can improve the UC induced by DSS. Their effect intensity is Indigo naturalis > indirubin > indigo based on disease activity index, body weight, colon length and pathological section. Indigo naturalis, indigo and indirubin also decrease the expression of NF-KB囚TLR4 and MYD88 proteins, thus reducing the level of related inflammation cytokines (IL-1 $\beta$, IL- 6 and TNF- $\alpha$ ) both in serum and tissue. In addition, Indigo naturalis and indigo improved symptoms of gut microbial disturbance, and decreased Firmicutes/Bacteroidetes ratio and the significantly increased probiotics such as Lactobacillus. Indirubin has little effect on the regulation of gut microbial.

Conclusions: Indigo naturalis could attenuate the DSS-induced UC in mice, by means of ameliorating intestinal inflammation, improving intestinal mucosa, and regulating the disturbed gut microbiota. Indigo and indirubin could also attenuate the DSS-induced UC in mice, but their comprehensive effect is not as good as Indigo naturalis.

\section{Background}

Ulcerative colitis (UC) is an inflammatory bowel disease (IBD) of unknown etiology, probably caused by a combination of genetic and environmental factors. Its morbidity is about $0.03 \%-0.1 \%$ worldwide, and is increasing in recent years. The precise etiology of UC is not known and cure of this disease is not yet possible so that the primary therapeutic goal is to relieve clinical symptoms and promote mucosal healing[1]. The classical drug for treating UC is 5-aminosalicylic acid, followed by short-term use of corticosteroids, immunomodulators and biologicals[2-4]. However, long term use of these drugs has some potential side effects, such as allergies, infections and anti-antibody reaction, etc[5]. It is thus of great significance and urgency to investigate the effective anti-UC drugs with a higher safety.

Complementary and alternative drugs are widely concerned by researchers due to their high safety and activity. Indigo Naturalis, a traditional Chinese medicine, is obtained from the extract of plants, such as Indigofera tinctoria, Isatis tinctoria, and Polygonum tinctorium. It has long been used to treat inflammatory diseases, including acute promyelocytic leukemia and psoriasis[6, 7]. Researches showed that indigo naturalis can improve the health of colitis model mice by regulating pro-inflammatory factors and improving the damage of colonic mucosa[8, 9]. A randomized, placebo-controlled trial has also been 
proved that indigo naturalis has an anti-UC activity[10]. This suggests that indigo naturalis may be a potential anti-UC drug. However, it is not clear for indigo naturalis what the mechanism and main active components in the treatment of UC.

The interaction of microorganisms in vivo determines the health of the body, especially in the colon where microorganisms are abundant[11]. Studies have shown that the intestinal mucosal inflammatory response caused by various factors is accompanied by the change of intestinal microecology and the imbalance of flora[12]. With the development of intestinal microecology research, intestinal flora regulators have become a hot research object in the treatment of UC. In this manuscript, the anti-UC effects and mechanisms of Indigo naturalis and its two main components (indigo and indirubin) were evaluated. Then their gut microbiota regulation was explored based on sequencing of 16S rDNA.

\section{Materials And Methods}

\section{Materials}

Indigo Naturalis (INN) was purchased by Sichuan Jiangyou Hengyuan Pharmaceutical Group Co., Ltd. It was identified by Professor Xu Run-chun, and deposited at the Chengdu University of Traditional Chinese Medicine, Chengdu, 611137 , China. Indigo Naturalis contained $2.38 \%$ indigo (IND) and $0.39 \%$ indirubin (INB) by HPLC. Standards of IND and INB were purchased from Chroma-Biotechnology Co., Ltd (Chengdu, China). Their purity is more than $98 \%$. Ethanol was purchased from Chengdu KeLong Chemical Factory (Chengdu, China). The enzyme-linked immunosorbent assay (ELISA) kits for TNF- $\alpha$, IL-1 $\beta$, IL- 6 and MPO were supplied by MultiScience (Lianke) Biotech Co., Ltd. Dextran sulfate sodium (DSS, 36-50 KDa) was purchased from MP Biomedicals (Aurora, USA). Sulfasalazine (SSZ) was purchased from Shanxi Tongda pharmaceutical Co., Ltd. BCA Protein Assay Kit and RIPA buffer were supplied Servicebio Biotechnology Co., Ltd (Wuhan, China).

\section{DSS-induced mice model}

Male BalB/c mice (6-8 weeks old, $20 \pm 2 \mathrm{~g}$ ) were obtained from Chengdu Dashuo Laboratory Animal Co., Ltd. (Permit No. SCXK (chuan) 2015-30, Chengdu, China). The animals were maintained under controlled conditions at temperature $20 \pm 0.5^{\circ} \mathrm{C}$, humidity $55 \pm 5 \%$ and with $12 \mathrm{~h} \mathrm{light/dark}$ cycles.

After one week of adaptation feeding, the mice were randomly divided into six groups $(n=6)$ : Normal, DSS induced Model, DSS with INN treatment, DSS with IND treatment, DSS with INB treatment and DSS with SSZ (positive drug) treatment. The UC mice model was established by drinking water containing DSS (3 \%,w/v) and the normal group received distilled water. After receiving DSS solutions for 5 days, the mice were orally administered with INN $(200 \mathrm{mg} / \mathrm{kg})$, IND $(4.76 \mathrm{mg} / \mathrm{kg})$ 区INB $(0.78 \mathrm{mg} / \mathrm{kg})$ and SSZ(200 $\mathrm{mg} / \mathrm{kg}$ ). Mice body weights were recorded every day during the entire experiment. 
The disease activity index (DAI) was recorded as the mean value of the following parameters: normal stool (0), soft stools (1), soft stools and slight bleeding (2), loose stools and slight bleeding (3), gross bleeding (4). At day 10, mice were sacrificed, the colons were measured and collected for histological analysis and protein extraction.

After 14 days of administration, all mice were taken blood from eyeballs. Then they were sacrificed by isoflurane inhalation, and their colon and feces samples were collected and exampled. Part of the colon segments were fixed by $4 \%$ paraformaldehyde, embedded in paraffin and then cut into $4 \mathrm{~mm}$ thick sections. These sections were stained with hematoxylin and eosin (HE) and periodic acid-Schiff (PAS), respectively, in accordance with the standard procedures for histopathological analysis. Additionally, part of colon segments was weighted and homogenized with 0.1Mphosphate buffer ( $\mathrm{pH} 7.4)$. The homogenate suspension was collected by centrifugation at 5,000 rpm for $15 \mathrm{~min}$. The amounts of inflammation-associated cytokines including IL $-1 \beta$, IL-6, TNF- $a$ and MPO were determined using ELISA kits according to the manufacturers' instructions.

\section{Western Blot Analysis}

The remaining colon segments were weighted and lysed in RIPA buffer with protease and phosphatase inhibitors on ice for $30 \mathrm{~min}$. The lysates were centrifuged at $12,000 \mathrm{~g}$ for $10 \mathrm{~min}$ at $4 \circ \mathrm{C}$ and the supernatant was collected as the total lysate protein. The protein concentration was determined with BCA Protein Assay Kit according to the manufacturers' instructions. The samples were boiled at $98{ }^{\circ} \mathrm{C}$ for 7 min, chilled on ice and separated using 10\% SDS-PAGE electrophoresis and then transferred to nitrocellulose membrane (Millipore Corporation, USA) at $25 \mathrm{~V}$ for overnight. The membrane was blocked in $5 \%$ no fat milk/TBST and incubated with primary antibody overnight at $4{ }^{\circ} \mathrm{C}$. The membrane was washed with TBST and subsequently incubated with an HRP conjugated secondary antibody that was detected using a chemiluminescent substrate.

\section{Effects on Gut Microbiota}

The normal, DSS, INN, IND and INB groups were selected for gut microbiota study. Total genomic DNAs from the feces of mice in various groups were extracted using DNeasy PowerSoil Kit (QIAGEN, Germany) according to the descriptions. Bacterial 16S rRNA gene sequences (V3-V4 region) were amplified by PCR instrument (Applied Biosystems R Gene Amp R PCR System 9700) using primer 5' $\rightarrow 3^{\prime}$ : 338F (5'ACTCCTACGGGAGGCAGCAG-3') and 806R (5'-GGACTACHVGGGTWTCTAAT-3'). The amplification procedures were conducted at $95^{\circ} \mathrm{C}$ for $3 \mathrm{~min}$ in the first place, and then followed by 30 cycles (denaturation at $95{ }^{\circ} \mathrm{C}$ for $30 \mathrm{~s}$, annealing at $54{ }^{\circ} \mathrm{C}$ for $30 \mathrm{~s}$, and elongation at $72{ }^{\circ} \mathrm{C}$ for $45 \mathrm{~s}$ ) and a final extension at $72{ }^{\circ} \mathrm{C}$ for $5 \mathrm{~min}$. PCR reactions were performed in triplicate: $20 \mu \mathrm{L}$ mixture containing $5 \mu \mathrm{L}$ 5×FastPfu Buffer, $2.5 \mu \mathrm{L}$ dNTPs $(2.5 \mathrm{mM}), 1 \mu \mathrm{L}$ Forward Primer $(5 \mu \mathrm{M}), 1 \mu \mathrm{l}$ Reverse Primer $(5 \mu \mathrm{M}), 0.5 \mu \mathrm{L}$ FastPfu Polymerase, $0.25 \mu \mathrm{L}$ BSA, $10 \mathrm{ng}$ template DNA and double-distilled water. Amplicons were 
extracted from $2 \%$ agarose gels, purified with the AxyPrepDNA Gel Extraction Kit (AXYGEN, USA) and then quantified with QuantiFluor ${ }^{\text {TM }}$-ST (Promega, USA).

The Illumina reads were sorted into different samples according to their barcoded index sequences. The paired-end reads were merged by FLASH and then assigned to each sample according to the unique barcodes, so as to get rid of the low-quality tags (length $<200 \mathrm{bp}$, more than two ambiguous bases ' $\mathrm{N}$ ', or the average base quality score $<30$ ). High-quality tags were clustered into operational taxonomic units (OTUs) using UPARSE algorithm in QIIME software based on a $97 \%$ sequence similarity, and these OTUs were further subjected to analysis using database of Greengenes by PyNAST algorithm[13]. Alpha and Beta diversities and principal coordinate analysis (PCOA) were analyzed by QIIME, Mothur, and R software. LEfSe analysis were done using Python LEfSe package[14].

\section{Statistical Analysis}

The experimental data were analyzed by GraphPad Prism 6.0 software and presented as mean \pm SD. The values of various groups were evaluated by one-way ANOVA and difference test. $P<0.05$ and $P<0.01$, calculated using SPSS software (version 21), were considered statistically significant.

\section{Results}

\section{the Healthy Condition of DSS-Induced UC in mice}

Preliminary experiments have shown that continuous administration of 3\% DSS in drinking water for more than three consecutive days could induce various symptoms, such as diarrhea and even hematochezia, which exhibit similarities with human UC. The body weight changes of the rats throughout the experiment clearly reflected their physiological status. Figure.1B shows a significant body weight loss of DSS-treated UC mice at day 10, in comparison with the normal group. However, rats in INN, IND, INB and SSZ groups exhibited a less distinct weight loss compared with the model group. In view of these representative clinical features, the DAI was used to evaluate the therapeutic activity of these drugs. As shown in Figure.1C, DAl scores of mice treated with DSS were significantly increased in comparison with those of the normal group. DAI scores of INN, IND, INB and SSZ groups remarkably dropped compared with the model group, indicating the improvement effect of these drugs on UC-related pathological states. Among of them, INN has the strongest improvement, followed by SZZ, INB and IND. In addition to the severe synechia, hyperemia, edema, the colon length of rats treated with DSS was apparently shortened. Figure.1D and E show the colon shortening of rats treated with DSS. Given all that, INN, IND and INB could significantly ameliorate the colon shortening.

\section{the Histopathological Changes in Colon Tissues}


The histopathological screening of mice colon sections by HE-staining revealed no necrosis or inflammation in the normal group (figure.2A). Mice in the DSS model group showed colon tissue injuries, including crypt distortion, goblet cell loss, severe epithelia damage, and mucosal inflammatory cell infiltration. However, the administration of INN, IND and INB could significantly protect colon crypt structure and reduce histologic inflammation. Moreover, it was hypothesized that INN, IND and INB could protect the intestinal mucosa from damages by reinforcing its self-repair. To further demonstrate this hypothesis, PAS-staining was conducted to investigate the intestinal mucosa changes after drugs administration during the DSS treatment. Figure.2B shows that DSS could remarkably decrease the expressions of mucins, which were involved in the repair of colon mucosa, compared with the normal group. Nevertheless, INN, IND, INB and SSZ could increase the mucin expressions, showing much more positive blue cells, especially IND. These results suggested that INN, IND and INB could inhibit colonic inflammation and promote the restoration of intestinal mucosa.

\section{the Changes of IL-1 $1 \beta, I L-6$, TNF- $\alpha$ and MPO both in Serum and tissue}

Inflammatory cytokines play important roles in the occurrence and progression of colitis. Serum and tissue levels of IL-1, IL-6, TNF and MPO were detected by Elisa kit. Levels of inflammatory cytokines IL$1 \beta \mathbb{Z} \mathrm{IL}-6$ and TNF-a both in serum and tissue were all increased significantly in DSS group compared with the normal group (Figure.3A, B and C). After intervening by INN, INB, IND, and SSZ, the levels of these cytokines declined, but they could not return to normal levels. IND and SSZ could significantly decrease the level of IL $-1 \beta$ in serum. IND and INB could reduce the level of IL-1 $\beta$ in tissue. All of administration groups could significantly decrease level of IL-6 both in serum and tissue. Conversely, all administration groups had little effect on TNF-a levels. MPO (myeloperoxidase) plays an important role in inflammation. Figure.3D showed that the level of MPO is less in tissue and higher in serum. The level of MPO both in serum and tissue significantly increased in DSS group compared with the normal group. INN and INB could return serum MPO levels to normal in DSS-induced mice.

the Expression of in TLR4, MyD88 and NF-KB (p65) Proteins

Compared with the normal group, DSS can significantly increase the expression of NF-KB (p65), TLR4 and MYD88 proteins (Figure.4). all the administration groups could decrease the expression of these proteins compared with DSS group. What's more, INB could return the expression of NF-KB (p65), TLR4 and MYD88 proteins to normal. INN could significantly decrease the expression of MYD88 proteins.

\section{the Structure Gut Microbiota in UC mice}

The expression of 16S rRNA gene sequence was used to evaluate the amelioration of INN, IND and INB on DSS-induced UC. The overall structure of gut microbiota was described and evaluated by $a$ and $\beta$ diversity analysis. Figure.5A showed that the number of OTUs in the sample is stable, and the amount of sequencing data can reflect the total number of OTUs in the sample. Figure.5B shows that the sample curve tends to be gentle and the distribution is relatively centralized, which shows that the amount of 
sequencing data is large enough to reflect the vast majority of microbial information in the sample. As shown in the Venn diagram (Figure.4C), 231 OTUs overlapped among these five groups, 300 OTUs between the normal and DSS groups, 340 OTUs between the DSS and INN groups, 341 OTUs between the DSS and IND groups, 340 OTUs between the DSS and INB groups. PCA (Figure.5D) and PCoA (Figure.5E) analyses based on the weighted UniFrac distance matrices suggested that the gut microbiota in three groups were significantly diversified. The non-metric multidimensional scale analysis (NMDS) based on $\beta$ diversity distance reflects the difference between different samples through the distance between points. The samples of normal, DSS, INN, IND and INB groups did not overlap (Figure.5F), indicating that there were significant differences in intestinal microbial composition between groups.

Histograms were used to reflect the differences among various groups on species and relative abundance of intestinal microbiota. At the phyla level (Figure.6A), Bacteroidetes, Firmicutes, Proteobacteria, Actinobacteria, and Tenericutes were the predominant and most numerous species in all groups. There were significantly increasing Bacteroidetes and decreasing Firmicutes in DSS groupcompared with the normal group. Some study reported that the increased level of Proteobacteria indicates the disorder of gut microbiota. Actually, there is little difference about Proteobacteria between the DSS group and the normal group and Proteobacteria in INN and IND groups significantly decreased. But Proteobacteria in INB group increased, indicating that there may be a disorder of gut microbiota. The detailed information about the regulation effect of INN, IND and INB on gut microbiota of UC mice was provided in bar plots at genus level (Figure.6B). Lactobacillus can ferment carbohydrates to produce lactic acid, which helps the human body digest and absorb, acidify the intestinal environment, prevent the adhesion of harmful bacteria in the intestinal epithelium and stimulate the production of immunoglobulin to enhance the host's immunity. Specifically, the increase in the abundance of Lactobacillus was observed in INN, IND and INB groups, indicating that INN, IND and INB could help to protect intestinal tract and alleviate intestinal inflammation by increasing the probiotics. Streptococcus bacteria are mostly conditional pathogens, and INN and IND could reduce the abundance of Streptococcus. Desulfovibrio is a sulfate-reducing bacterium that has been reported to produce hydrogen-sulfate that damages intestinal epithelial cells and induces mucosal inflammation[15]. INN and INB could restore the abundance of Desulfovibrio to normal, indicating that INN and INB, indicating INN and INB could protect intestinal tract.

Lefse (LDA effect size) was used to determine the bacterial genera with significant differences among groups. Figure.6C and $\mathrm{D}$ indicates that there are five special groups in the normal group, one in the model group, three in the INN group, eight in the IND group and seven in the INB group. Lefse method is a combination of non-parametric test and linear discriminant analysis, which is suitable for the test of microbial abundance difference. The LDA value is greater than 3.5 as the screening criterion to determine dominant microorganisms in this group. Roseburia, Alloprevotella, Prevotellaceae, Lachnospiraceae_UCG_001 are dominant microorganisms in the normal group. Anaerotruncus is dominant microorganism in DSS group. The dominant microorganisms in the INN group were _Eubacterium_fissicatena_group and Butyricimonas. The dominant microorganisms in the IND group were Coprococcus_3, Ruminiclostridium_6, and_Ruminococcus_torques_group. The dominant 
microorganisms in INB group were Rhodospirillaceae, Rhodospirillales, Alphaproteobacteria, Prevotellaceae_UCG_001, Proteobacteria and Rhodospirillaceae_g_uncultured.

\section{Discussion}

Inflammatory bowel disease (IBD), including UC and Crohn's disease, is a chronic inflammatory bowel disease. In recent years, many studies have shown that UC is associated with intestinal mucosal barrier damage, genetic susceptibility and environmental factors[16, 17]. Clinical treatment UC is usually treated with anti-inflammatory or immunosuppressive drugs that have the disadvantages of relapse after discontinuation, long-term adverse drug reactions, and unsatisfactory results in some refractory cases[18]. Other studies have proved that INN, IND and INB can improve UC, but their therapeutic mechanism is not clear[19-21].

TLR4 / MyD88 / NF - K B pathway is one of the most important signaling pathways activated or inhibited in the pathogenesis of UC. TLR4, the first member of TLR family, belongs to type I transmembrane receptor of TLR interleukin superfamily. It can promote inflammation and regulate immune response. What's more, it can recognize and combine pathogen related molecular pattern, activate NF - KB-related protein pathway, and promote inflammation[22]. MyD88 is an important linker protein in the TLR4 signal transduction process. TIR region of its C-terminal binds to the TIR region of TLR4[23], while its N-terminal acts as a death region and can bind to NF-KB, which promotes the inflammatory factor Released in large quantities, causing an immune response in the intestinal mucosa[24]. NF-KB is closely related to the transcription of inflammatory transmitters, cytokines and other genes. Binding to the $\kappa B$ site, it initiates an inflammatory response, which breaks the immune balance in the intestine and finally leads to the occurrence of UC[25]. In this manuscript, INN, IND and INB can inhibit the activation of TLR4 / MyD88 /

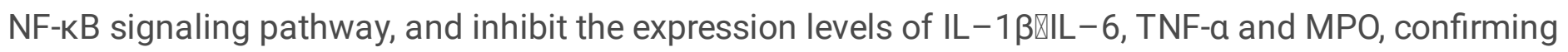
that INN, IND and INB can play a role in UC treatment by regulating the expression of TLR4 / MyD88 / NF$\mathrm{KB}$ signaling pathway and related cytokines.

Recently, a large number of studies have focused on the relationship between gut microbiota disorders and ulcerative $\$ and the intestinal microbiota has been demonstrated as one of the key factors to influence nutrient metabolism and immune response, and keep the host healthy in various intestinal diseases[26, 27]. The gut microbiota mainly consists of Firmicutes, Bacteroidetes, Actinobacteria and Proteobacteria, which account for more than $98 \%$ of the total gut microflora[28]. The reduction of intestinal microflora diversity and low abundance of Firmicutes were observed in IBD patients compared with healthy man[29, 30]. There are reports both in increase and decrease of the abundance of Proteobacteria and Bacteroidetes, which may be related to the actual situation of IBD patients[31]. In this study $\llbracket$ based on the 16S rRNA gene sequence analysis of samples from the normal group, the DSS-induced UC group》 respective INN $\mathbb{I N D}$ and INB treatment groups, the predominant intestinal bacteria profiles were greatly diversified. Figure. 6 show that the DSS-induced UC is often accompanied by shifts in gut microbiota structure, with a significant decrease of intestinal bacterial diversity, a reduction of Firmicutes and an increase of Bacteroidetes amounts. Further analysis found that Anaerotruncus,is a probiotic and 
dominant microorganism in DSS-induced group. This result is contrary to the previous reports, which may be caused by the stress reaction of the body[32,33]. In addition, INN, IND and INB have different regulation on structure of gut microbiota in UC mice. The dominant bacteria in INN and INB groups are probiotics, such as Butyricimonas, Eubacterium, Coprococcus and Ruminococcus. Interestingly, the dominant species in INB group almost belong to Proteobacteria, whose abundance increasing means the occurrence of flora disorder. Although INB can improve the condition of UC, it has a weak regulation of intestinal flora.

\section{Conclusion}

INN, IND and INB can improve DSS-induced UC via inhibiting TLR4 / MyD88 / NF - K B pathway and regulating gut microbiota. Among them, the comprehensive effect of INN is the strongest, followed by INB and IND. In addition, INB has a weak regulation of intestinal flora.

\section{List Of Abbreviations}

UC: ulcerative colitis

INN: Indigo Naturalis

INB: indirubin

DAl: disease activity index

PAS: periodic acid-Schiff
IBD: inflammatory bowel disease

IND: indigo

\section{Declarations}

\section{Ethics approval and consent to participate}

This study was conducted in strict accordance with the recommendations of the Guidelines for the Care and Use of Laboratory Animals of the Ministry of Science and Technology of China. The protocol and experimental designs were approved by the Ethical Committee of Chengdu University of Traditional Chinese Medicine (Approval ID:2017BL-003). All possible steps were taken to avoid the animals' suffering at any stage of the experiment. At the end of study, the animals were sacrificed following anesthesia with isoflurane inhalation.

\section{Consent for publication}

The manuscript is approved by all authors for publication.

\section{Availability of data and material}

The datasets used in this study are available from the corresponding author upon reasonable request. 


\section{Competing interests}

The authors declare no competing financial interests.

\section{Funding}

We are grateful to the support of National Key Research \& Development Program (2018YFC1707205) and National Natural Science Foundation Project (81773918).

\section{Author contributions}

Zhang DK, Wang F, Yang QY, He YN and Han L designed the study; He YN, Ma LL, Xu RC and Li N performed experiments; Zhang DK, Wang F and Yang QY analyzed data; He YN and Yang QY supplied materials and analytic tools; Yang QY, He YN and Ma LL wrote the paper.

\section{Acknowledgements}

We thank Dr. Zhang Chen for guiding the experiment.

\section{References}

[1] Harbord, M., Eliakim, R., Bettenworth, D., et al. Third European Evidence-based Consensus on Diagnosis and Management of Ulcerative Colitis. [J]. J Crohns Colitis, 2017, 11: 769-84.

[2] Ford, A.C., Achkar, J.P., Khan, K.J., et al. Efficacy of 5-aminosalicylates in ulcerative colitis: systematic review and meta-analysis [J]. Am J Gastroenterol, 2011, 106: 601-16.

[3] Kornbluth, A., Sachar, D.B. Ulcerative colitis practice guidelines in adults: American College Of Gastroenterology, Practice Parameters Committee [J]. Am J Gastroenterol, 2010, 105: 501-23; quiz 24.

[4] Rogler, G. Top-down or step-up treatment in Crohn's disease? [J]. Dig Dis, 2013, 31: 83-90.

[5] Renna, S., Cottone, M., Orlando, A. Optimization of the treatment with immunosuppressants and biologics in inflammatory bowel disease [J]. World J Gastroenterol, 2014, 20: 9675-90.

[6] Yang, M.H., Wan, W.Q., Luo, J.S., et al. Multicenter randomized trial of arsenic trioxide and RealgarIndigo naturalis formula in pediatric patients with acute promyelocytic leukemia: Interim results of the SCCLG-APL clinical study [J]. Am J Hematol, 2018, 93: 1467-73.

[7] McDermott, L., Madan, R., Rupani, R., et al. A Review of Indigo Naturalis as an Alternative Treatment for Nail Psoriasis [J]. J Drugs Dermatol, 2016, 15: 319-23.

[8] Xiao, H.T., Peng, J., Hu, D.D., et al. Qing-dai powder promotes recovery of colitis by inhibiting inflammatory responses of colonic macrophages in dextran sulfate sodium-treated mice [J]. Chin Med, 2015, 10: 29. 
[9] Wang, Y., Liu, L., Guo, Y., et al. Effects of indigo naturalis on colonic mucosal injuries and inflammation in rats with dextran sodium sulphate-induced ulcerative colitis [J]. Exp Ther Med, 2017, 14: 1327-36.

[10] Naganuma, M., Sugimoto, S., Mitsuyama, K., et al. Efficacy of Indigo Naturalis in a Multicenter Randomized Controlled Trial of Patients With Ulcerative Colitis [J]. Gastroenterology, 2018, 154: 935-47.

[11] Franzosa, E.A., Sirota-Madi, A., Avila-Pacheco, J., et al. Gut microbiome structure and metabolic activity in inflammatory bowel disease [J]. Nat Microbiol, 2019, 4: 293-305.

[12] Kostic, A.D., Xavier, R.J., Gevers, D. The microbiome in inflammatory bowel disease: current status and the future ahead [J]. Gastroenterology, 2014, 146: 1489-99.

[13] Edgar, R.C. UPARSE: highly accurate OTU sequences from microbial amplicon reads [J]. Nat Methods, 2013, 10: 996-8.

[14] Segata, N., Izard, J., Waldron, L., et al. Metagenomic biomarker discovery and explanation [J]. Genome Biol, 2011, 12: R60.

[15] Rowan, F., Docherty, N.G., Murphy, M., et al. Desulfovibrio bacterial species are increased in ulcerative colitis [J]. Dis Colon Rectum, 2010, 53: 1530-6.

[16] Lin, Z., Zu, X.P., Xie, H.S., et al. Research progress in mechanism of intestinal microorganisms in human diseases [J]. Acta pharmaceutica Sinica, 2016, 51: 843-52.

[17] Tatiya-Aphiradee, N., Chatuphonprasert, W., Jarukamjorn, K. Immune response and inflammatory pathway of ulcerative colitis [J]. J Basic Clin Physiol Pharmacol, 2018, 30: 1-10.

[18] Yamamoto, T., Carvello, M., Lightner, A.L., et al. Up-to-date surgery for ulcerative colitis in the era of biologics [J]. Expert Opin Biol Ther, 2020, 20: 391-8.

[19] Tokuyasu, N., Shomori, K., Amano, K., et al. Indirubin, a Constituent of the Chinese Herbal Medicine Qing-Dai, Attenuates Dextran Sulfate Sodium-induced Murine Colitis [J]. Yonago Acta Med, 2018, 61: 12836.

[20] Kawai, S., lijima, H., Shinzaki, S., et al. Indigo Naturalis ameliorates murine dextran sodium sulfateinduced colitis via aryl hydrocarbon receptor activation [J]. J Gastroenterol, 2017, 52: 904-19.

[21] Liang, Y.N., Yu, J.G. Indigo Naturalis Ameliorates Dextran Sulfate Sodium-Induced Colitis in Mice by Modulating the Intestinal Microbiota Community [J]. Molecules, 2019, 24.

[22] Burgueno, J.F., Abreu, M.T. Epithelial Toll-like receptors and their role in gut homeostasis and disease [J]. Nat Rev Gastroenterol Hepatol, 2020.

[23] Nagpal, K., Plantinga, T.S., Wong, J., et al. A TIR domain variant of MyD88 adapter-like (Mal)/TIRAP results in loss of MyD88 binding and reduced TLR2/TLR4 signaling [J]. J Biol Chem, 2009, 284: 25742-8. 
[24] Feng, J., Guo, C., Zhu, Y., et al. Baicalin down regulates the expression of TLR4 and NFkB-p65 in colon tissue in mice with colitis induced by dextran sulfate sodium [J]. Int J Clin Exp Med, 2014, 7: 4063-72.

[25] Xu, X., Zhang, L., Liu, Z., et al. Therapeutic Efficacy of the Traditional Chinese Medicine Baishaoqiwu on TNBS-induced Colitis is Associated with Down-regulation of the TLR4/MyD88/NF-kappaB Signaling Pathway [J]. In Vivo, 2016, 30: 181-6.

[26] Khan, I., Ullah, N., Zha, L., et al. Alteration of Gut Microbiota in Inflammatory Bowel Disease (IBD): Cause or Consequence? IBD Treatment Targeting the Gut Microbiome [J]. Pathogens, 2019, 8.

[27] Stallmach, A., Grunert, P., Pieper, D., et al. [Ulcerative colitis: Does the modulation of gut microbiota induce long-lasting remission?] [J]. Z Gastroenterol, 2019, 57: 834-42.

[28] Alam, M.T., Amos, G.C.A., Murphy, A.R.J., et al. Microbial imbalance in inflammatory bowel disease patients at different taxonomic levels [J]. Gut Pathog, 2020, 12: 1.

[29] Ricciuto, A., Sherman, P.M., Laxer, R.M. Gut microbiota in chronic inflammatory disorders: A focus on pediatric inflammatory bowel diseases and juvenile idiopathic arthritis [J]. Clin Immunol, 2020, 215: 108415.

[30] Walker, A.W., Sanderson, J.D., Churcher, C., et al. High-throughput clone library analysis of the mucosa-associated microbiota reveals dysbiosis and differences between inflamed and non-inflamed regions of the intestine in inflammatory bowel disease [J]. BMC Microbiol, 2011, 11: 7.

[31] Nishida, A., Inoue, R., Inatomi, O., et al. Gut microbiota in the pathogenesis of inflammatory bowel disease [J]. Clin J Gastroenterol, 2018, 11: 1-10.

[32] Dong, L., Li, J., Liu, Y., et al. Toll-like receptor 2 monoclonal antibody or/and Toll-like receptor 4 monoclonal antibody increase counts of Lactobacilli and Bifidobacteria in dextran sulfate sodiuminduced colitis in mice [J]. J Gastroenterol Hepatol, 2012, 27: 110-9.

[33] Lin, Y., Zheng, X., Chen, J., et al. Protective Effect of Bruguiera gymnorrhiza (L.) Lam. Fruit on Dextran Sulfate Sodium-Induced Ulcerative Colitis in Mice: Role of Keap1/Nrf2 Pathway and Gut Microbiota [J]. Front Pharmacol, 2019, 10: 1602.

\section{Figures}




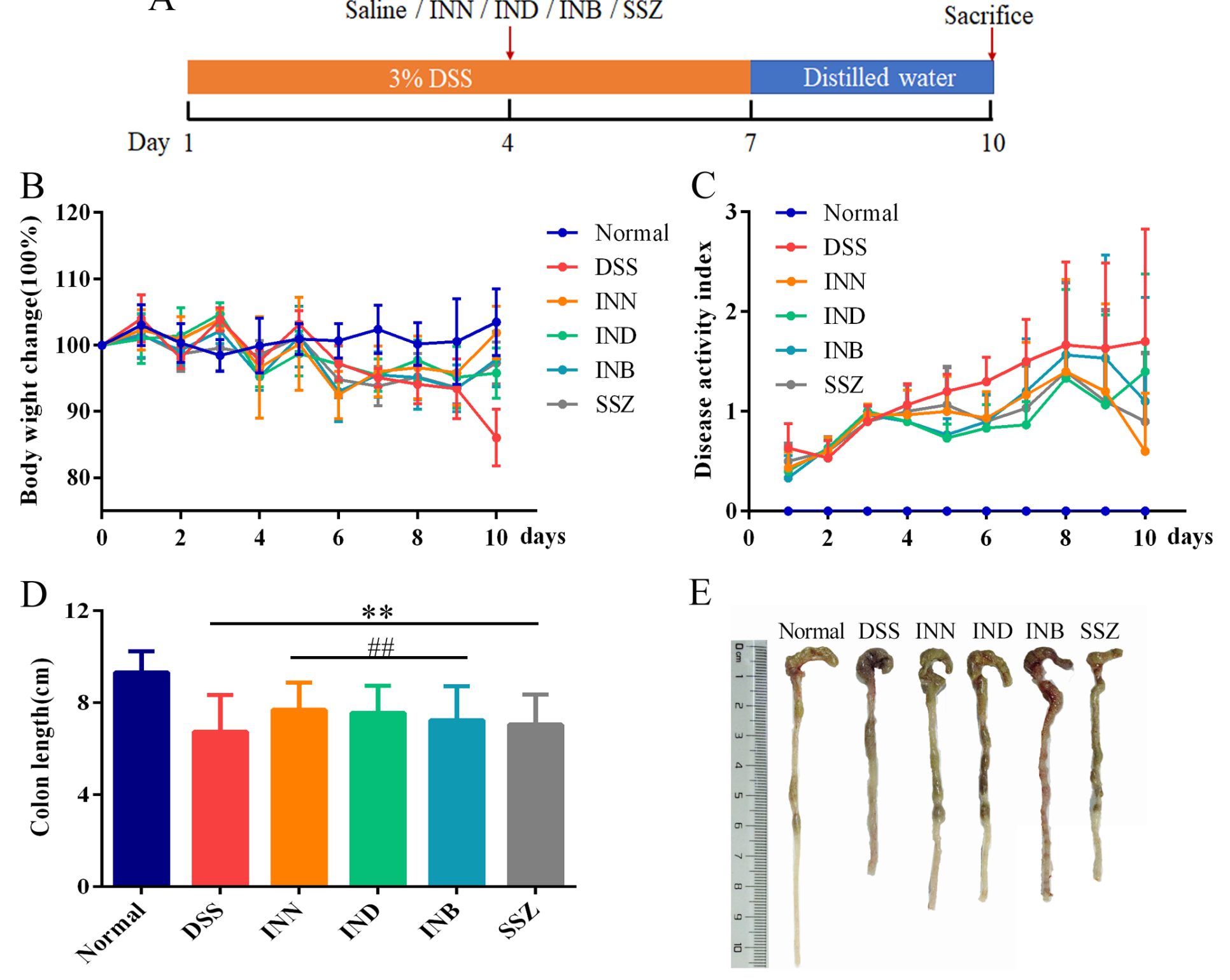

Figure 1

Effects of INN, IND, INB and SZZ treatment on the of DSS-induced UC mice after 7 days of continuous gavage. (A) The medication regimen; (B) body weight change in various groups; (C) disease activity index in various groups; $(D)$ colon length in various groups; $(E)$ photos of colon length in various groups. Vs normal group, ${ }^{\star *} P<0.01$; Vs DSS group, \#\#P<0.01. Data are expressed as mean $\pm S D(n=6)$. 

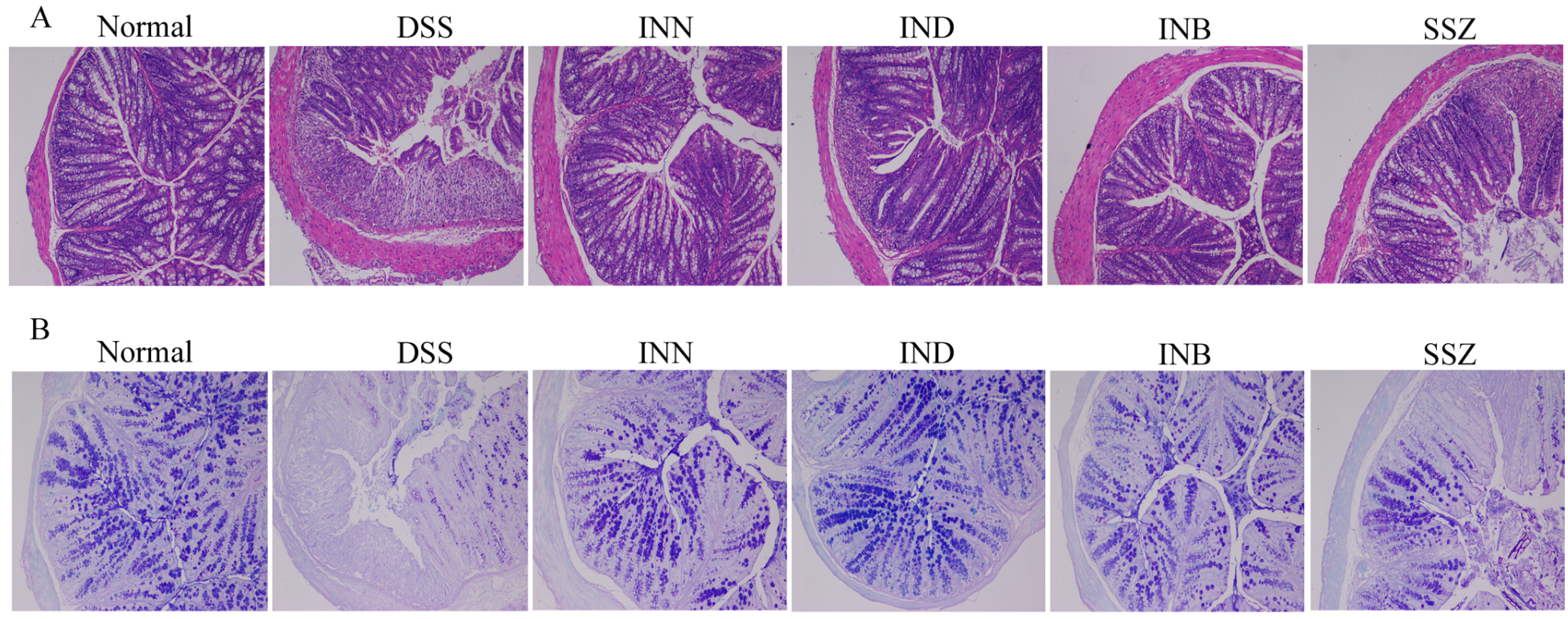

DSS
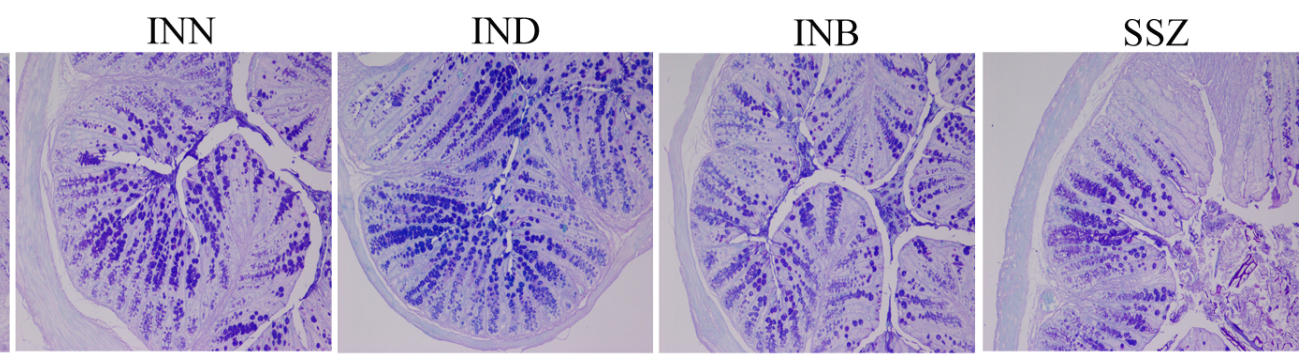

Figure 2

Histological sections of colonic tissue stained with HE (A) and PAS (B) (magnification囚100) 

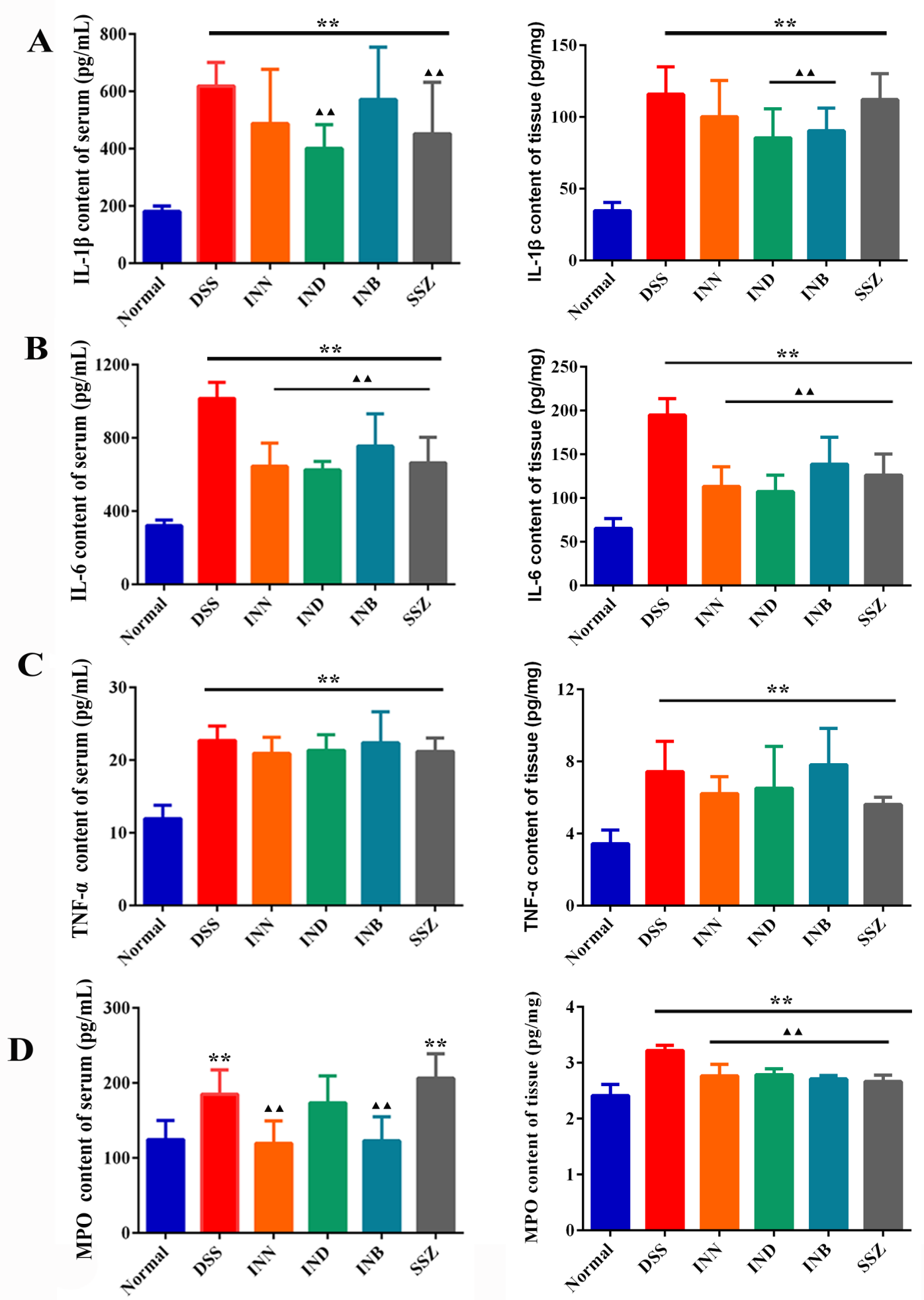

Figure 3

The Changes of IL-1 $\beta(A)$, IL-6 (B), TNF-a (C) and MPO (D) both in Serum and tissue. **P < 0.01; Vs DSS group, $\boldsymbol{\Delta} \Delta P<0.01$. Data are expressed as mean $\pm S D(n=6)$. 

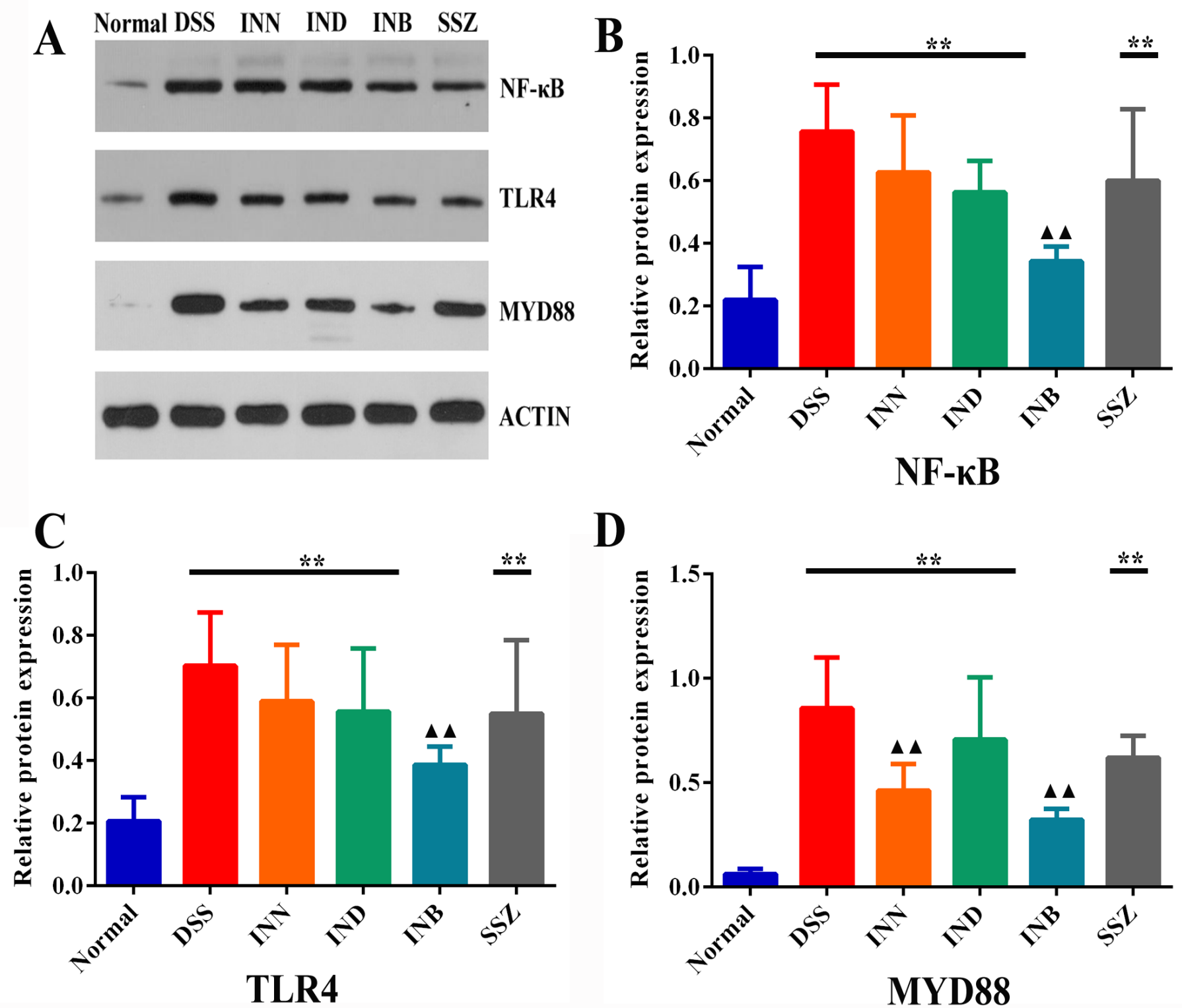

D

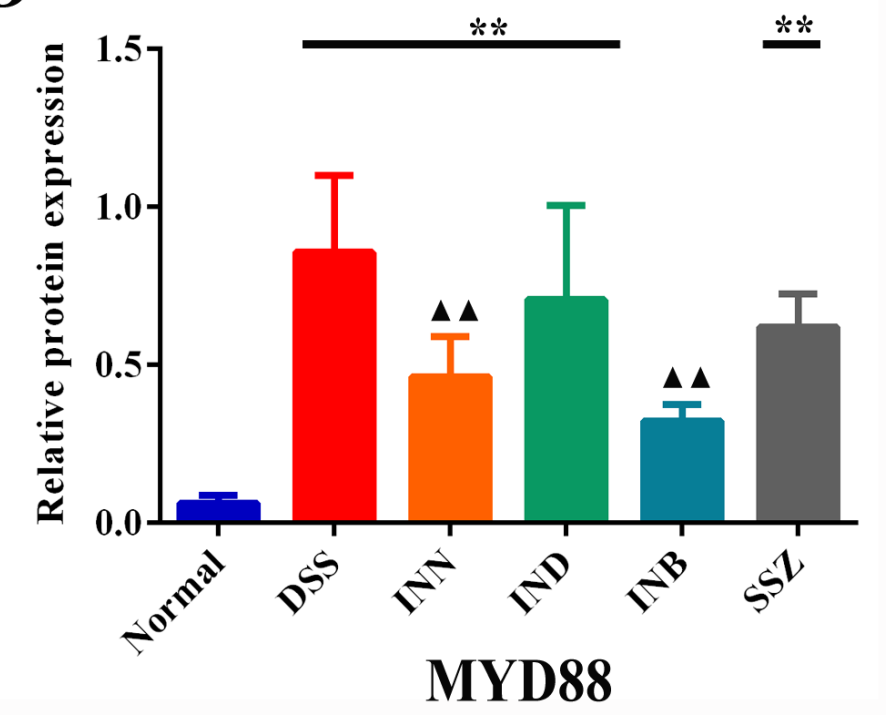

Figure 4

The Expression of in NF-kB, TLR4 and MyD88 proteins (A)expression of NF-kB, TLR4 and MyD88 proteins was tested using Western blot analysis; (B) relative expression of NF-KB; (C) relative expression of TLR4; (D) relative expression of MYD88. $* * P<0.01$; Vs DSS group, $\mathbf{\Delta} \mathbf{\Delta} P<0.01$. Data are expressed as mean \pm $\operatorname{SD}(n=6)$. 

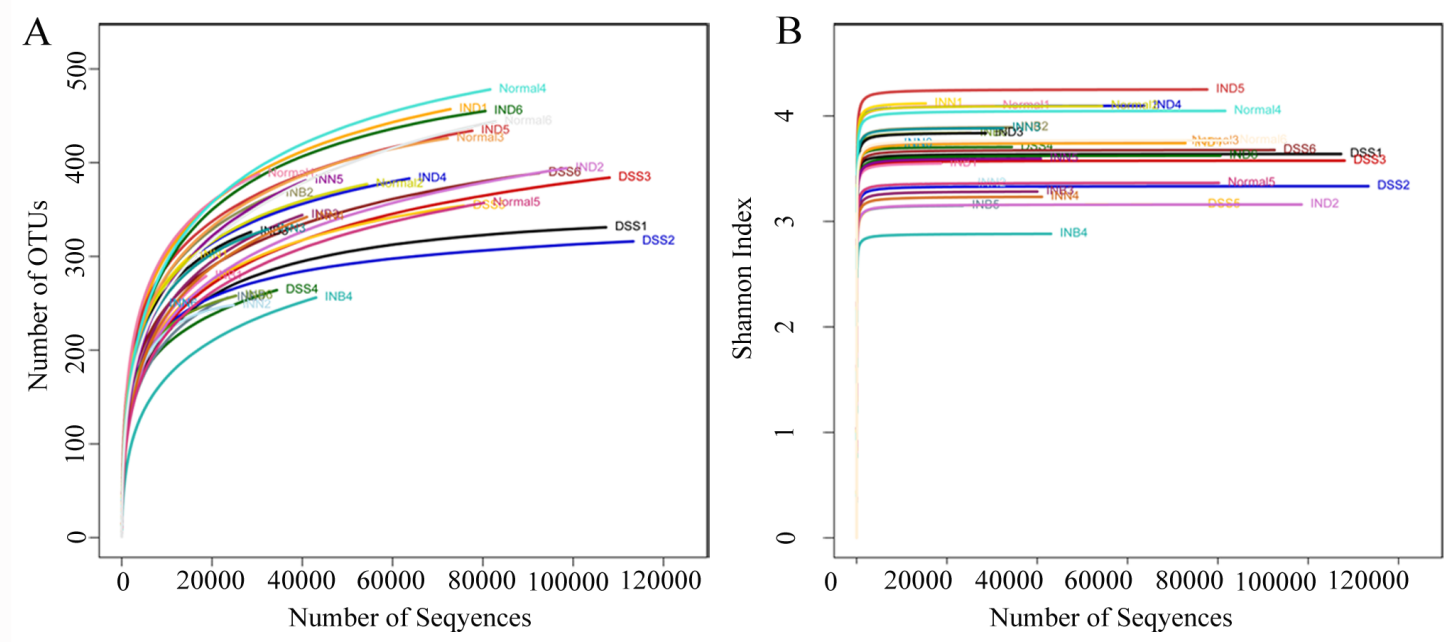

$\mathrm{C}$

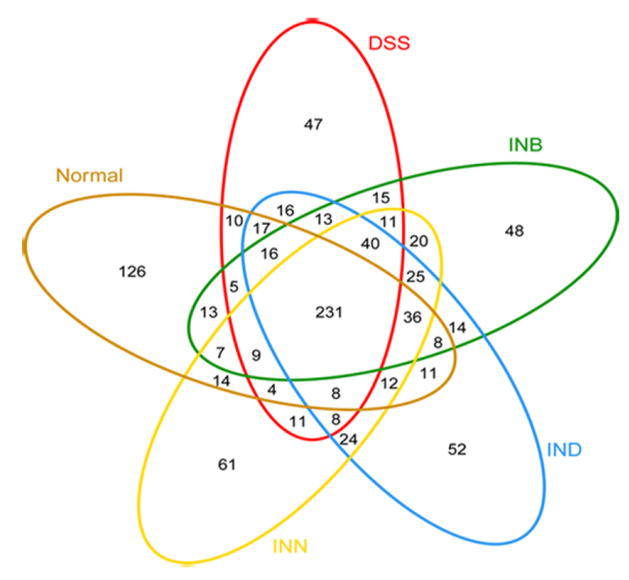

$\mathrm{D}$
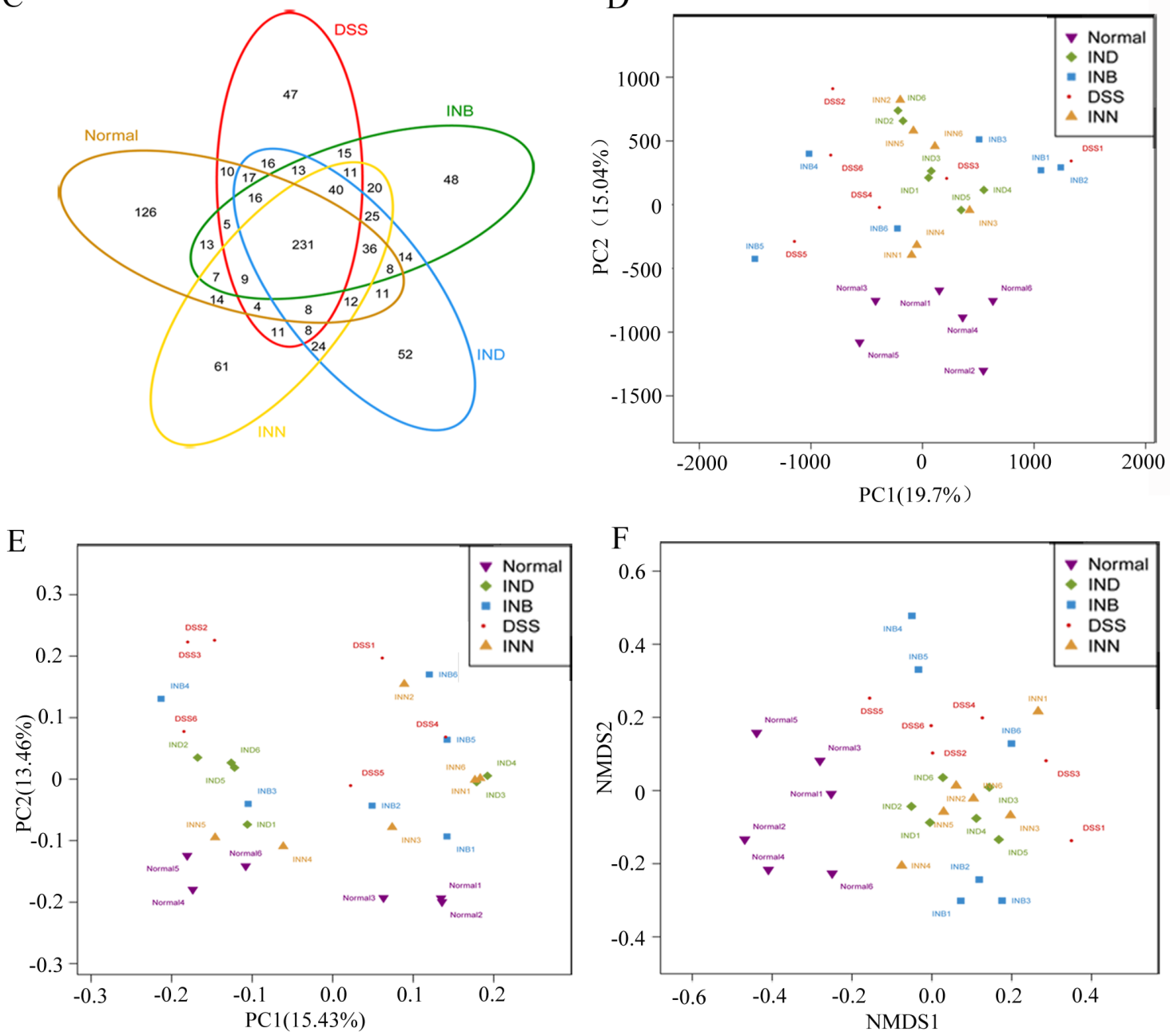

\section{Figure 5}

INN, IND and INB regulation on the disturbed gut microbiota in DSS-induced UC rats. (A) rarefaction curves determined at the $97 \%$ similarity level; (B) shannon-Wiener curves of samples; (C) venn diagram of OTU in the five groups; (D) multiple sample PCA analysis; (E) multiple sample PCoA analysis; (F) Multiple sample NMDS analysis. 


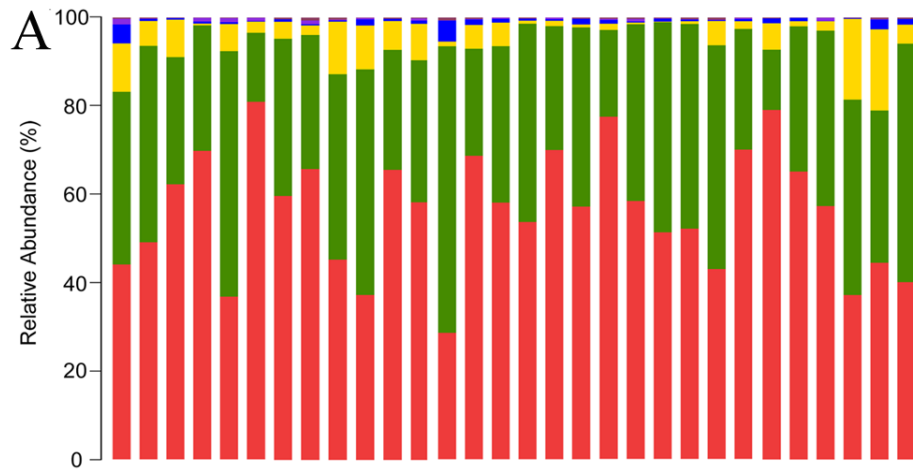

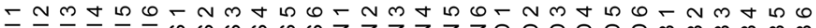

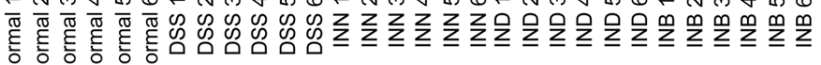
$z<z z z$

$\square$ Bacteroidetes
$\square$ Firmicutes

$\square$ Proteobacteria $\square$ Tenericutes

- Actinobacteria $\square$ Others
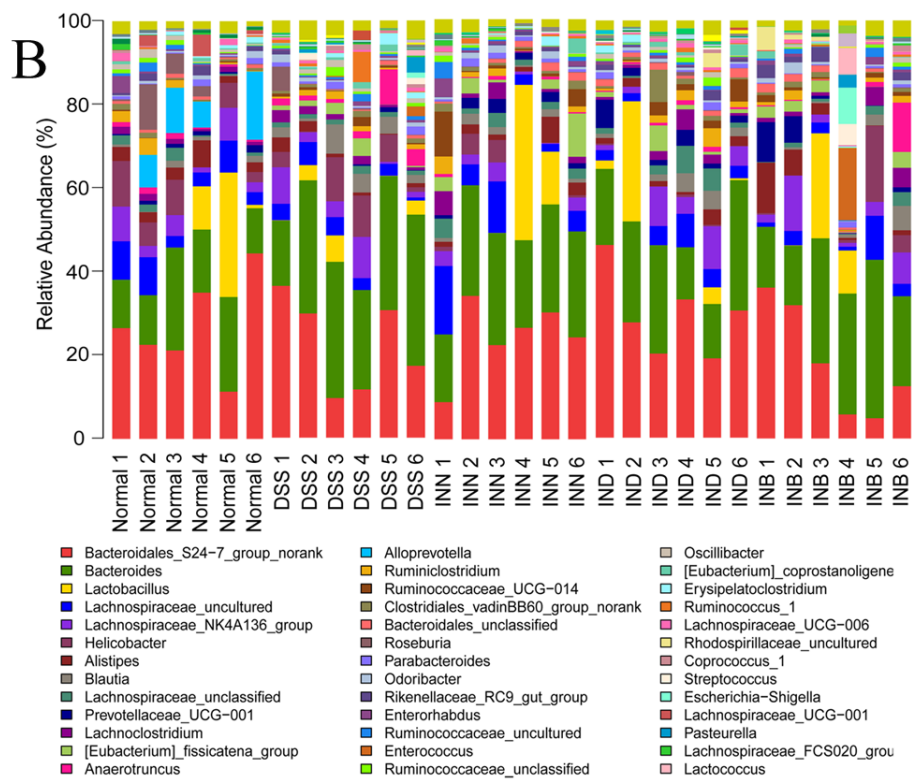

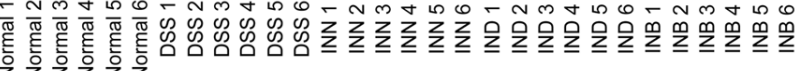

Anaerotruncus

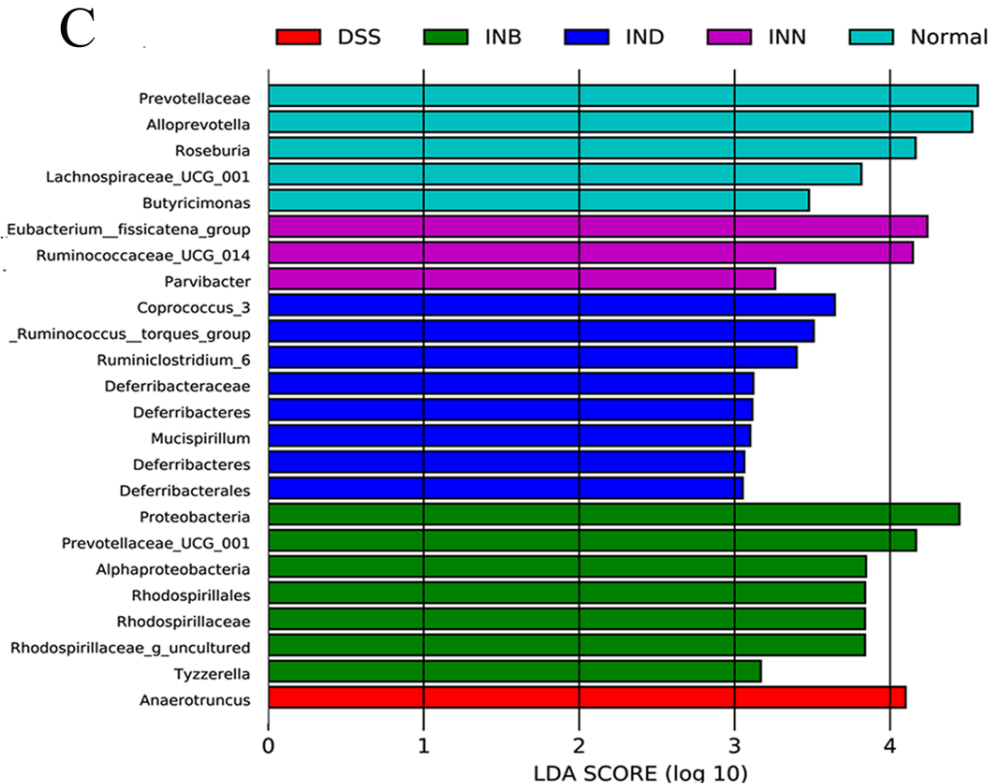

$\mathrm{D}$ Cladogram

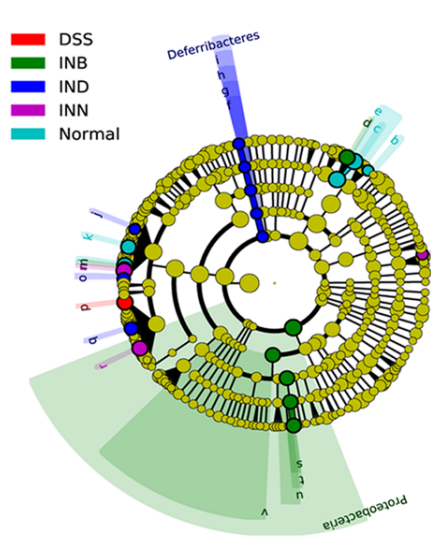

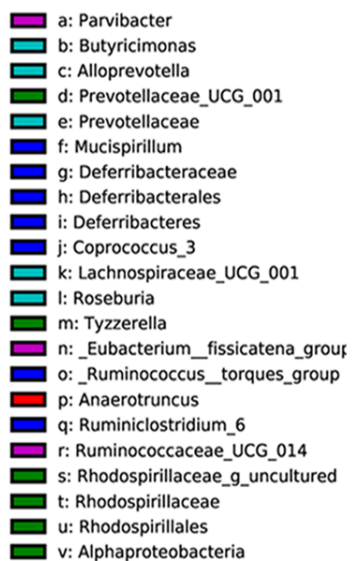

Figure 6

Gut microbial community structures of rats from various groups and difference in dominant

microorganisms between groups. (A) Microbial community bar plot by phylum; (B) Microbial community bar plot by genus; (C) Distribution histogram based on Lefse; (D)Cladogram. $(n=6)$ 